\title{
Synthesis of fluorescence labelled aptamers for use as low-cost reagents in HIV/AIDS research and diagnostics
}

\author{
KANYANE MALATJI $^{1,2}$, PASCALINE N. FRU ${ }^{2}$, HAZEL MUFHANDU ${ }^{3}$ and KABAMBA ALEXANDRE ${ }^{1}$ \\ ${ }^{1}$ Council for Scientific and Industrial Research, Emerging Research Area Platform, Next Generation Health Cluster, \\ Pretoria, Gauteng 0001; ${ }^{2}$ Department of Surgery, School of Clinical Medicine, Faculty of Health Sciences, \\ University of the Witwatersrand, Johannesburg $2193 ;{ }^{3}$ Department of Microbiology, North West University, \\ School of Biological Sciences, Mmabatho, North West 2735, South Africa
}

Received June 1,2021; Accepted September 20, 2021

DOI: 10.3892/br.2021.1491

\begin{abstract}
Aptamers are nucleic acids selected by systematic evolution of ligands by exponential enrichment. They have potential as alternatives to antibodies in medical research and diagnostics, with the advantages of being non-immunogenic and relatively inexpensive to produce. In the present study, gp120 aptamers conjugated with fluorescein isothiocyanate (FITC) were generated, which could interact with HIV-1 gp120. A previously isolated gp120 aptamer, CSIR 1.1, was conjugated with FITC by incubation with 1-ethyl-3-(3-dimethylaminopropyl) carbodiimide and imidazole. The conjugation and binding to the glycoprotein were confirmed by flow cytometry. FITC conjugated aptamers showed an increase in fluorescence emission 24-fold higher than baseline, and this difference was statistically significant $(\mathrm{P}=0.0016)$. Compared with a commercially available biotinylated anti-gp120 antibody, detected using FITC conjugated streptavidin, the emission of fluorescence obtained from the FITC-conjugated aptamer was 8 -fold higher, suggesting a stronger interaction with gp120. In addition, the FITC conjugated aptamer neutralized HIV-1 pseudoviruses with an average $\mathrm{IC}_{50}$ of $21.3 \mathrm{nM}$, similar to the parent aptamer that had an $\mathrm{IC}_{50}$ of $19.2 \mathrm{nM}$. However, the
\end{abstract}

Correspondence to: Dr Kabamba Alexandre, Council for Scientific and Industrial Research, Emerging Research Area Platform, Next Generation Health Cluster, Building 20, Meiring Naude Road, Pretoria, Gauteng 0001, South Africa

E-mail: aalexandre@csir.co.za

Dr Hazel Mufhandu, Department of Microbiology, North West University, School of Biological Sciences, 1090 Corner of Dr Albert Luthuli and University Drive, Building A12-B, Mmabatho, North West 2735, South Africa

Email: hazel.mufhandu@nwu.ac.za

Abbreviations: FITC, fluorescein isothiocyanate; SELEX, systematic evolution of ligands by exponential enrichment

Key words: CSIR 1.1, anti- human immunodeficiency syndrome-1 gp120, RNA aptamers, flow cytometry, capture assay difference in inhibition between the two aptamers was not statistically significant $(\mathrm{P}=0.784)$. These results indicate that the FITC-conjugated aptamer generated in the present study could potentially be used as a low-cost reagent in HIV/AIDS research and diagnostics.

\section{Introduction}

At present, there are $\sim 38.0$ million people living with HIV/AIDS globally, with the annual number of deaths standing at 700,000 in $2019(1,2)$. Although HIV/AIDS affects all parts of the world, infections are highest in developing countries. Africa remains the most severely affected region, with the number of infected people standing at 25.7 million in 2019 (2). South Africa has the highest number of infected individuals, with 7.5 million people infected (1). Therefore, it remains critical to continue with HIV research to reduce the burden of disease, especially in countries that are the most affected.

HIV has an RNA genome surrounded by p24 capsid proteins that can be detected in the blood during infection (3). The virus also comprises a p17 matrix and the protease, reverse transcriptase and integrase enzymes used during viral replication in host cells (4). The viral envelope consists of a trimeric complex made of the heterodimer proteins gp120 and gp41, which make up the gp160 protein. These proteins are essential for recognition by target cells and infection $(5,6)$. More specifically, the gp41 protein is responsible for fusing the virus and cellular membranes, while gp120 binds to CD4 receptors expressed on host cell surfaces (6).

Antibodies are used in flow cytometry for the detection of cells infected with HIV-1. These include antibodies specific to gp120 and those against p24 $(3,6)$. However, these reagents have several disadvantages. For example, antibodies are produced by the immunization of animals with the antigen of interest; as a result, they are expensive to make, and their manufacture is time consuming (7). In addition, target molecules that do not elicit an immune response cannot be detected using antibodies $(8,9)$. Furthermore, antibodies are large, non-flexible, cannot be modified easily with functional groups and are not stable, as they are redox-sensitive to $\mathrm{pH}$ and temperature (8). These limitations can be circumvented using aptamers that are cheaper, considerably smaller and can be generated against almost any target molecule. 
Aptamers are short, synthetic single-stranded nucleic acid (DNA or RNA) oligomers. They fold into unique 3D structures by intramolecular interactions. These structures are characterized by stems, loops, bulges, hairpins, pseudoknots, and triplexes or quadruplexes that allow them to bind with high affinity and specificity to different target molecules $(10,11)$. Aptamers are isolated from a random single stranded-library using a technique called systematic evolution of ligands by exponential enrichment (SELEX) in as little as 5-9 rounds of selection (10). SELEX employs a repetitive process of selection and amplification of high binding oligonucleotides, which results in the exponential increase of the best-binding aptamers $(12,13)$. The number of selection rounds in this technique depends on the diversity of the nucleic acid library, target features and concentration, ratio of target molecules to oligonucleotides, the stringency of selection and bias of amplification $(14,15)$. Previously, aptamers have been selected against HIV-1 gp120 to inhibit its interaction with the CD4 receptor (11). Some aptamers have been reported to inhibit or neutralize HIV-1 infection with 50\% inhibition concentration $\left(\mathrm{IC}_{50}\right)$ values in the nanomolar range $(10,11)$. Aptamers are known to be equivalent to antibodies in their potential as research, diagnostic and therapeutic reagents $(16,17)$. Their advantages over antibodies also include their lack of immunogenicity and ease of modification by chemical substitutions $(7,18)$. Together, these advantages make aptamers attractive molecules for diagnostic purposes and suitable for the replacement of antibodies in several research and therapeutic uses.

Although aptamers are attractive molecules, they have several limitations. RNA and DNA molecules are unstable in cells or sera; however, this has been circumvented by chemically modifying the aptamer for stability and nuclease resistance $(10,19)$. For example, it has been shown that disulfide cross-links can be introduced into the nucleic acid secondary structure, endowing significant increases in thermal stability without compromising the structural integrity (20). The addition of 2'flouropyrimidine groups to aptamers during selection has been reported to increase the stability and protection against nucleases (10). Furthermore, chemical modification increases not only the stability, but also the half-life of aptamers (11).

The use of fluorescence-labelled aptamers has been explored for application as detectors and inhibitors of target molecules. For example, a fluorescence-labelled 5'-hexachlorofluorescein pseudoknot RNA aptamer and a 5'-carboxyfluorescein DNA aptamer have been isolated against HIV-1 reverse transcriptase $(21,22)$. These aptamers were intended for use as inhibitors of the viral reverse transcriptase. RNA and DNA aptamers conjugated to fluorescent dyes have also been used as reagents to detect or diagnose cancer and viral infections $(23,24)$.

Based on the above studies, it was hypothesized that the conjugation of FITC to CSIR1.1 and UCLA1 aptamers, specific to HIV-1 gp120, will not alter the aptamers' structures, binding affinity and specificity for gp120. This will enable them to be used as low-cost reagents in HIV-1 research and diagnostics that can benefit resource-poor settings. Here, the labelling of HIV-1 envelope specific aptamers to the fluorescent dye FITC and the subsequent testing of these aptamers for potential use in applications involving flow cytometry and virus capture assays is reported. The results showed that the dye conjugated better with the aptamer that had no stability related modifications at its 5' or 3' ends. In addition, the conjugated aptamer could detect the recombinant HIV-1 gp120 coated on latex beads by flow cytometry. It could also detect the glycoprotein in its natural environment when expressed on the viral envelope. Lastly, this aptamer performed better in its detection of HIV-1 gp120 when compared with an antibody-based detection system.

\section{Materials and methods}

Reagents. The UCLA1 anti-gp120 RNA aptamer, a shortened derivative (54 nucleotides in length) of the B40t77 aptamer $(11,25)$, and the CSIR1.1 anti-gp120 RNA aptamer, made up of 112 nucleotides, were used in the present study. UCLA1 was obtained from the University of California, Los Angeles (California, USA). The CSIR1.1 aptamer was isolated at the Council for Scientific and Industrial Research in Pretoria, South Africa, by London et al (10). Both aptamers were previously shown to have anti-HIV inhibitory activity $(10,11)$. Plasmids encoding HIV-1 envelopes ZM53M. PB12, CAP239.2.00.G3J, CAP210.E8 and DU156.12 were obtained from the AIDS Virus Research Unit of the National Institute for Communicable Diseases (Johannesburg, South Africa). Biotinylated gp120 polyclonal antibody, used as a positive control, was purchased from Abcam (cat no. ab53937).

Cell lines used for the neutralization assay. Two types of cell lines were used, TZM-bl and 293 T cells. The TZM-bl cells used for the neutralization assays were obtained from the National Institutes of Health HIV/AIDS Reference Reagent Program (niaid.nih.gov/research/nih-aids-reagent-program). TZM-bl cells are HeLa cell clones engineered to express, CD4 receptors, and CCR5 and CXCR4 co-receptors. These cells also contain integrated reporter genes for firefly luciferase regulated by HIV-1 long terminal repeat (11). The $293 \mathrm{~T}$ cells, used for transfection to make pseudotyped viruses, were obtained from the American Type Culture Collection. They are adherent cells originally derived from human embryonic kidney cells. Both TZM-bl and 293T cell lines were sub-cultured at a concentration of $1 \times 10^{6}$ cells $/ \mathrm{ml}$ every 2 days using DMEM (Thermo Fisher Scientific, Inc.) supplemented with 10\% FBS (Thermo Fisher Scientific, Inc.), with gentamycin (Thermo Fisher Scientific, Inc.) as the antibiotic at $37^{\circ} \mathrm{C}$, with $5 \% \mathrm{CO}_{2}$ and $95 \%$ humidity.

Pseudoviruses for neutralization and capture assays. The pseudoviruses used in the present study were generated as previously described (11). The Env-pseudotyped virus stocks were produced for ZM53M.PB12, CAP210.E8, CAP239.G3J and DU156.12 viruses and the 50\% tissue culture infective doses $\left(\mathrm{TCID}_{50}\right)$ were quantified as previously described (26).

Conjugation of FITC to anti-gp120 UCLAI and CSIR 1.1 aptamers

Using a commercially available kit. FITC is a fluorophore that contains reactive isothiocyanate and carboxylic acid groups that react with nucleophiles such as amines. This fluorophore has a molecular weight of $389.38 \mathrm{Da}$. The anti-gp120 UCLA1 and CSIR1.1 aptamers to FITC, and the FluoroTag ${ }^{\mathrm{TM}}$-FITC 


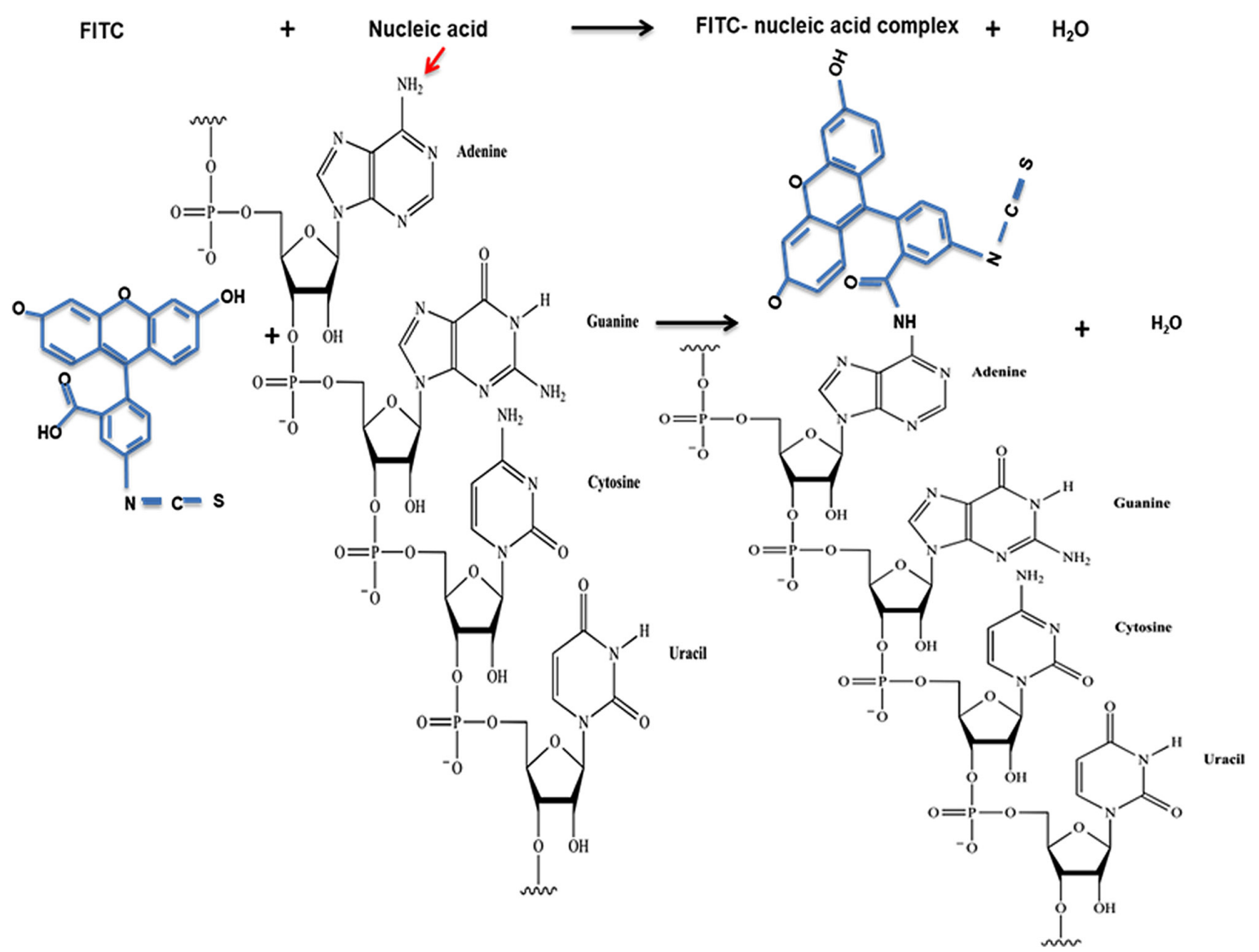

Figure 1. The possible reaction between FITC and a nucleic acid (RNA) at the 5' amine group. The FITC molecule is blue, and the reactive amine on the nucleic acid is also indicated. The reaction was expected to occur between the carboxyl group of FITC and the amine group on the 5' end of the nucleic acid. FITC, fluorescein isothiocyanate.

conjugation kit (Sigma-Aldrich; Merck KGaA) were used to conjugate the aptamers according to the manufacturer's protocol. Briefly, a $0.1 \mathrm{M}$ sodium carbonate-bicarbonate buffer, $\mathrm{pH}$ 9.4, was prepared according to the manufacturer's instructions. FITC was reconstituted in $2 \mathrm{ml}$ buffer. Then $89 \mu 1$ anti-gp120 UCLA1 and CSIR1.1 aptamers dissolved in the same buffer were each added to a $1.5 \mathrm{ml}$ Eppendorf tube, and the volume made up to $200 \mu \mathrm{l}$ with the buffer. This was followed by a dropwise addition of $50 \mu \mathrm{l}$ FITC and shaking for $2 \mathrm{~h}$ in the dark at room temperature.

Using an in-house method. To conjugate UCLA1 and CSIR1.1 aptamers to FITC, $1,750 \mathrm{ng} / \mu 1$, in $7.5 \mu 1$, of these compounds were mixed with $1.25 \mathrm{mg}$ 1-ethyl-3-(3-dimethylaminopropyl) carbodiimide and $0.25 \mathrm{M}(5 \mu \mathrm{l})$ FITC in imidazole $(0.1 \mathrm{M}, \mathrm{pH} 6)$. The mixture was thoroughly vortexed and centrifuged at $17,000 \mathrm{xg}, 15^{\circ} \mathrm{C}$ for $5 \mathrm{~min}$. To the reaction, $20 \mu 10.1 \mathrm{M}, \mathrm{pH} 6$ imidazole was added and mixed using a shaker at room temperature for $48 \mathrm{~h}$. This was followed by centrifugation of each conjugated aptamer in 3,000 and 10,000 molecular weight cut-off (MWCO) Sartorius vivaspin columns (Sigma-Aldrich; Merck KGaA) to remove unconjugated FITC. The different MWCO columns were used for both aptamers to compare their respective efficiency in eliminating unconjugated FITC. The retained aptamers were washed three times with $0.1 \mathrm{M}$ imidazole by centrifuging for $20 \mathrm{~min}$ at $3,901 \mathrm{x} \mathrm{g}$, $15^{\circ} \mathrm{C}$. The washed solution was collected and stored at $-20^{\circ} \mathrm{C}$. The two aptamer solutions were transferred to different wells of a 96-well black plate, and fluorescence was measured using the DTX 880 Multimode detector at a wavelength of $485 \mathrm{~nm}$. Unconjugated FITC was used as the positive control.

Expected reaction between FITC and aptamer. The FITC isothiocyanate and carboxyl groups are reactive groups that react with nucleophiles, such as amines and hydroxyls (27). A free hydroxyl group is found on the 3' end of nucleic acids when the sequence terminates with an unmodified nucleotide. In contrast, a free amine is found at the $5^{\prime}$ end when it has an unmodified purine or pyrimidine (Fig. 1). Of note, in a nucleic acid molecule, there are also amine groups attached to different bases (28). However, these are unlikely to react with FITC due to steric hindrance caused by their location within the RNA molecule. The inability of outside molecules to react with bases within nucleic acids due to steric hindrance has been previously described (29-31).

\section{Detection of fluorescence emitted by FITC-conjugated UCLAI and CSIR1.1 aptamers}

Fluorescence microscopy. Fluorescence microscopy imaging was performed using TZM-bl cells infected with HIV-1 ZM53M.PB12 to evaluate whether the conjugation was successful. Cells were cultured in the presence of DEAE-dextran in a flat-bottom 96-well plate at a concentration of 10,000 cells/well/100 $\mu$ l DMEM supplemented with $10 \%$ FBS and gentamicin to make them more susceptible to infection. This was followed by infection with $200 \mathrm{TCID}_{50}$ of 
ZM53M.PB12 pseudovirus. The reaction was incubated for $48 \mathrm{~h}$ at $37^{\circ} \mathrm{C}, 5 \% \mathrm{CO}_{2}$ and $95 \%$ humidity.

The infected cells were stained with the FITC-conjugated anti-gp120 UCLA1 aptamer before imaging using the Cytation 3 Cell Imaging Multimode reader (BioTek Instruments, Inc.).

Flow cytometry. The conjugation of the anti-gp120 CSIR1.1 aptamer to FITC was further confirmed using flow cytometry. Latex beads (Sigma-Aldrich; Merck KGaA) were diluted to a $1 \%$ working stock solution in double-distilled water. Then, $10 \mu 1$ recombinant gp120 at a concentration of $1.29 \mathrm{mg} / \mathrm{ml}$ was added to $4 \mu 11 \%$ latex beads, and the solution was incubated overnight at $4{ }^{\circ} \mathrm{C}$, followed by washing the beads coupled to gp120 and blocking twice with $500 \mu 10.1 \%$ BSA-PBS using centrifugation for $2 \mathrm{~min}$ at $17,000 \mathrm{x} \mathrm{g}, 4^{\circ} \mathrm{C}$. The beads were then resuspended in $700 \mu 1$ the $0.1 \%$ BSA-PBS buffer. The beads were then transferred to flow cytometry tubes, centrifuged at $3,901 \times \mathrm{g}, 4^{\circ} \mathrm{C}$ for $5 \mathrm{~min}$ and resuspended in $100 \mu \mathrm{l}$ $1 \mathrm{X}$ BD binding buffer (Becton-Dickinson and Company). A total of 4 test tubes were used with gp120 coated (or coupled) beads stained with a 3 -fold serial dilution of FITC-conjugated CSIR1.1 aptamer starting at $869 \mathrm{nM}$. The control tubes contained gp120 coated beads stained with FITC only, starting at $869 \mathrm{nM}$, uncoated beads stained with FITC-conjugated CSIR1.1 aptamer and other uncoated beads stained with FITC only. Fluorescence was subsequently measured using the BD LSR Fortessa cell analyzer (Becton-Dickinson and Company) across the FITC channel with an excitation of $485 \mathrm{~nm}$, and analyzed using the BD FACSDiva version 9.0 (BD Biosciences). The percentage fluorescence was calculated relative to the concentration of the FITC and FITC-conjugated aptamer $(869 \mu \mathrm{M})$ using FlowJo version 10 (FlowJo, LLC) analysis with unstained beads as a reference point. This was performed after instrument voltage optimization to exclude electronic noise and background.

Capture assay. This assay was used to determine the ability of CSIR1.1 FITC-conjugated anti-gp120 aptamer to bind gp120 on whole HIV-1 particles and was performed as previously described (32) with some modifications. As CSIR1.1 aptamer has been shown to bind to gp120 (10), $100 \mu 1 \mathrm{gp} 41$ 10E8 monoclonal antibody (mAb) at a concentration of $1 \mu \mathrm{g} / \mathrm{ml}$ was used in this assay to avoid competitive binding between the capturing antibody and the aptamer. Thus, a high-binding 96-well black plate was coated with $1 \mu \mathrm{g} / \mathrm{ml}$ anti-gp41 10E8 and incubated at $4{ }^{\circ} \mathrm{C}$ overnight. The plate was then washed and blocked with $3 \% \mathrm{BSA}$, and $50 \mu 1 \mathrm{HIV}-1$ pseudoviruses were added and incubated for $1 \mathrm{~h}$ at $37^{\circ} \mathrm{C}$, with $5 \% \mathrm{CO}_{2}$ and $95 \%$ humidity to allow binding to $10 \mathrm{E} 8 \mathrm{mAb}$. Pseudovirus ZM53. PB12, CAP210.E8 and Du156.12 were used as they are known to bind the CSIR1.1 aptamer strongly (10). After incubation, the viruses were washed with PBS followed by the addition of a 3-fold serial dilution of FITC-conjugated CSIR1.1 aptamer, with an initial concentration of $50 \mu \mathrm{g} / \mathrm{ml}$. Biotinylated gp120 antibody and FITC-conjugated streptavidin were used as positive controls at a starting concentration of $50 \mu \mathrm{g} / \mathrm{ml}$, and the negative control was the unconjugated aptamer. The plates were incubated again as above and then washed. Next, fluorescence was measured at $485 \mathrm{~nm}$ using the DTX 880 multimode detector (Beckman Coulter, Inc.). The excitation wavelength was $485 \mathrm{~nm}$, and emission occurred at $535 \mathrm{~nm}$. SoftMaxPro version 6.4 (Molecular Devices, LLC) was used for data acquisition and analysis.

\section{HIV-1 infectivity assay}

TZM-bl neutralization assay. The single-cycle neutralization assay was used to test the infectivity of the pseudovirus CAP239.G3J and the inhibitory effect of the aptamer (CSIR1.1), as previously described (33). This assay used a 3 -fold dilution series of the inhibitor (CSIR1.1 FITC-conjugated aptamer or the CSIR1.1 unconjugated aptamer). The dilution series started with an $11.9 \mathrm{nM}$ concentration of each aptamer, followed by the addition of $50 \mu 1$ HIV-1 CAP239.G3J pseudovirus. The negative control wells included uninfected and untreated TZM-bl cells. Luminescence readings at $510 \mathrm{~nm}$ were performed using the TECAN Infinite F500 Luminometer and i-control 1.5 software (Tecan Group, Ltd.). The $\mathrm{IC}_{50}$ value of the aptamers was calculated as the inhibitory concentration that caused a 50\% reduction in relative light units compared to the virus control (without inhibitor) after subtraction of the background (without virus and inhibitor).

Statistical analysis. Statistical analysis was performed using SPSS version 22 (IBM Corp.) and GraphPad Prism version 5.02 (GraphPad Software, Inc.) using a one-way ANOVA with a post-hoc Tukey's test, or an unpaired Student's t-test with 95\% confidence. Each experiment was repeated three times, and data are expressed as the mean \pm standard deviation. Graphs were constructed using GraphPad Prism version 5. $\mathrm{P}<0.05$ was considered to indicate a statistically significant difference.

\section{Results}

Conjugation of FITC to gp120 aptamers using two different methods. CSIR1.1 and UCLA1, which have been previously shown to bind to the gp120 glycoprotein, was conjugated with FITC to generate a fluorescence-labelled gp120 aptamer. This was achieved using a commercially available kit and an in-house method. As shown in Fig. 2A, the conjugation of the dye using the kit was more successful in the case of CSR1.1. The fluorescence intensity for this aptamer after conjugation increased from 0 to $6 \times 10^{6} \mathrm{RFU}$. At the same time, with UCLA1, the value was very low at $4 \times 10^{5}$, resulting in the difference of fluorescence emitted by the two conjugated aptamers to be $5.6 \times 10^{6}$. The fluorescence conjugation using the in-house method was successful for both CSIR1.1 and UCLA1, although the former still emitted significantly more fluorescence than the latter (Fig. 2B). The difference in the fluorescence emitted by the two aptamers was $\sim 2$-fold. Given that the stoichiometry of the aptamer and FITC reaction was 1:1 for CSIR1.1 and UCLA1, these results suggested that the former was more efficient in interacting with FITC than the latter.

Next, the fluorescence emission of FITC conjugated gp120 aptamer was compared with unconjugated FITC to determine the percentage of the dye in the solution that became conjugated to the aptamer after the conjugation reaction. The in-house conjugation method was used for the CSIR1.1 aptamer in this experiment, as it was the condition that emitted the highest fluorescence. It should also be noted that in all subsequent experiments in the present study, only FITC-conjugated CSIR1.1 was used because of its high fluorescence emission. 

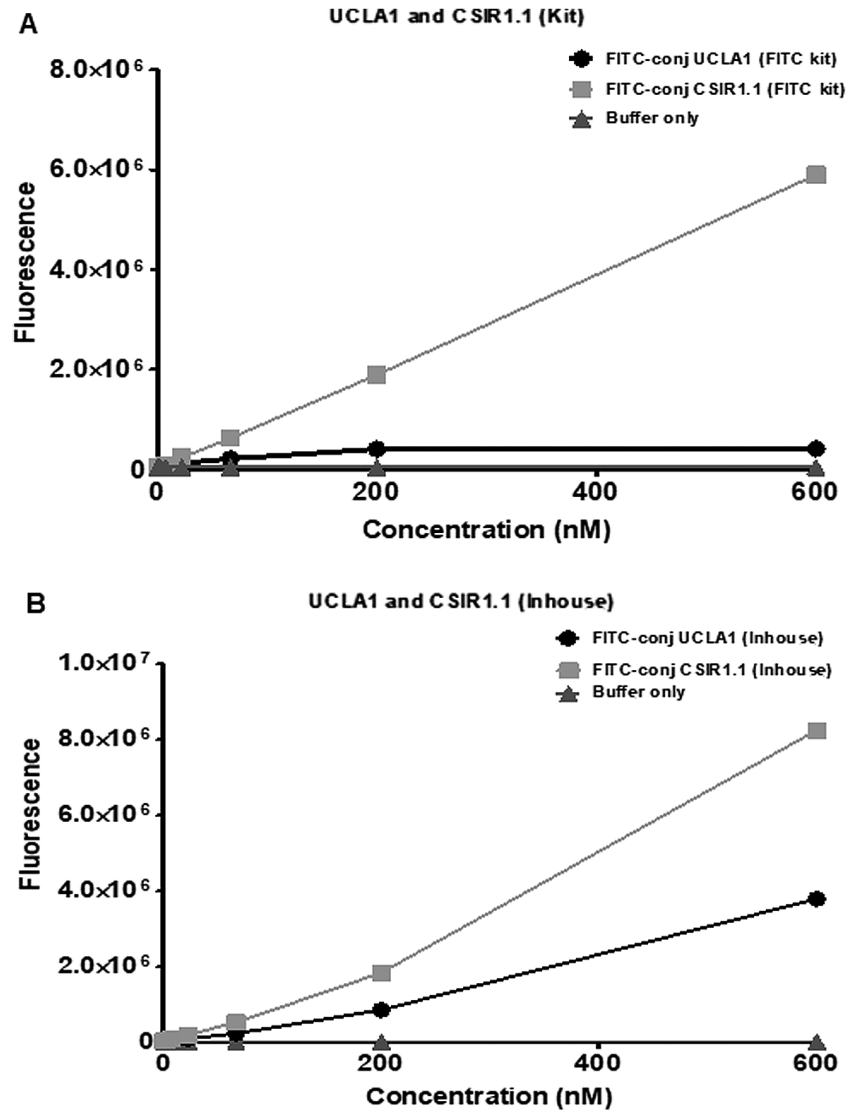

Figure 2. Fluorescence emission of UCLA1 and CSIR1.1 aptamers conjugated to FITC. CSIR1.1 and UCLA1 were coupled with FITC using (A) a commercially available kit; or (B) an in-house method developed at theCouncil for Scientific and Industrial Research. The results shown represent the mean \pm standard deviation of three experiments repeated on different days. FITC, fluorescein isothiocyanate.

Here, a $0.25 \mathrm{M}$ concentration of FITC was conjugated with CSIR1.1 before filtration, using a Vivaspin column to remove the dye that did not react with the aptamer. Then the fluorescence emitted by the resulting FITC-CSIR 1.1 conjugate was compared to that emitted by the same amount of FITC used in the conjugation reaction, which was used as the control. As shown in Fig. 3, at any given concentration of FITC reacted with the aptamer, the amount of fluorescence emitted by FITC-CSIR1.1 was only $20 \%$ of that obtained from FITC only. These data suggest a $20 \%$ efficiency of the conjugation reaction between FITC and CSIR1.1. The efficiency of this reaction was lower $(\sim 1 \%)$ with the UCLA1 aptamer (Fig. S1).

Detection of the interaction between FITC-conjugated CSIRI.1 and HIV-1 gp120 by flow cytometry. Since one of the possible applications of FITC-conjugated CSIR1.1 is its use in flow cytometry to detect HIV-1 infected and gp120-expressing cells (34), its binding to the glycoprotein was assessed using this technique. This was undertaken by coupling latex beads with recombinant gp120 derived from the Consensus C virus, a representative of all circulating HIV-1 subtype C viruses (35). The gp120 coupled beads were incubated with the FITC-conjugated CSIR1.1 aptamer (36). As the negative control, the beads (uncoupled) were also stained with FITC (37). Other controls included unstained gp120-coupled

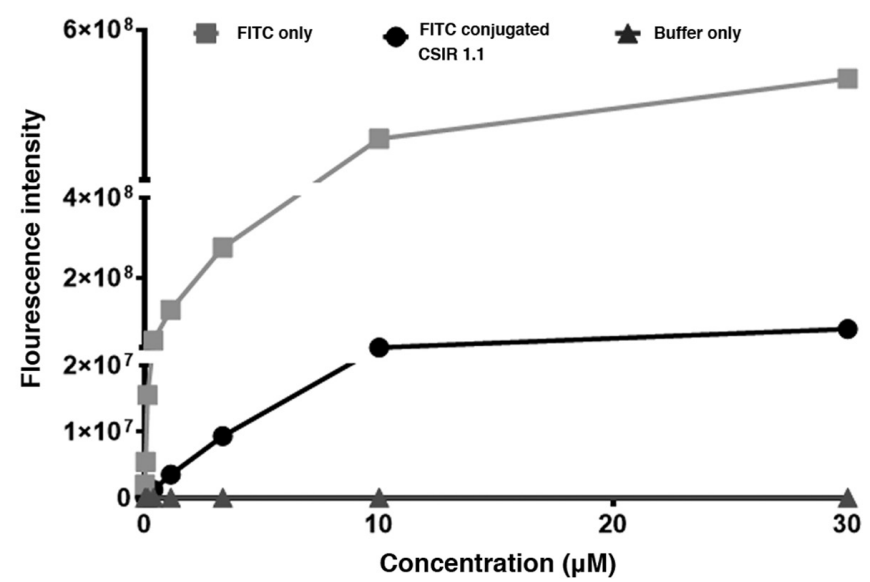

Figure 3. Comparison of fluorescence emission between FITC-CSIR1.1 and unconjugated FITC. A known amount of FITC was reacted with CSIR1.1. After removing unconjugated dye, the FITC-CSIR1.1 fluorescence was compared to fluorescence emitted by the same amount of FITC used in the reaction. The results shown represent the mean \pm standard deviation of three experiments repeated on different days. FITC, fluorescein isothiocyanate.

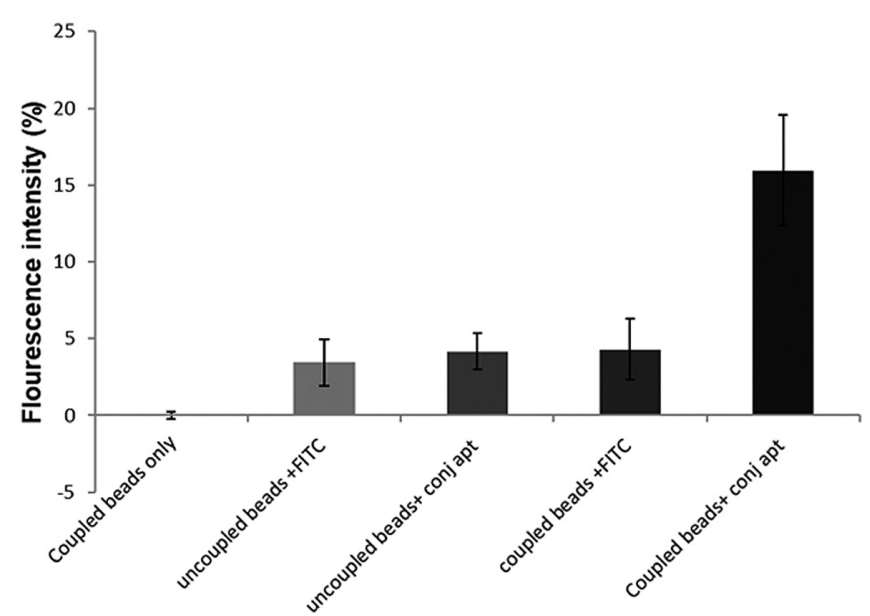

Figure 4. Binding of FITC-conjugated CSIR1.1 to gp120 on latex beads Quantitative bar graph of the flow cytometry data representing the percentage of the mean fluorescence (relative fluorescence unit) $(n=3)$, and the error bars indicate standard deviation. The HIV-1 gp120 coupled beads stained with FITC-CSIR1.1 exhibited significantly higher fluorescence compared with the uncoupled beads stained with the same conjugated aptamer. $\mathrm{P}=0.016$. FITC, fluorescein isothiocyanate.

beads and uncoupled beads incubated with the FITC-CSIR1.1. After acquiring 10,000 events on the flow cytometer, no fluorescence was observed with the unstained gp120-coupled beads. Only a $3.5 \pm 1.51 \%$ increase in fluorescence emission was reported for the gp120-uncoupled beads stained with FITC alone. Uncoupled beads with the FITC-conjugated CSIR1.1 showed a $4.14 \pm 1.18 \%$ increase in fluorescence emission (Figs. 4 and S2). These findings suggest some background level/autofluorescence due to the interaction between the dye and the beads. However, more importantly, a $16 \pm 3.6 \%$ increase in fluorescence was reported for gp120-coupled beads stained with FITC-CSIR1.1 (Figs. 4 and S2).

Binding of FITC-conjugated CSIR1.1 to gp120 on intact HIV-1 particles. Given that the gp120 coupled on latex beads 


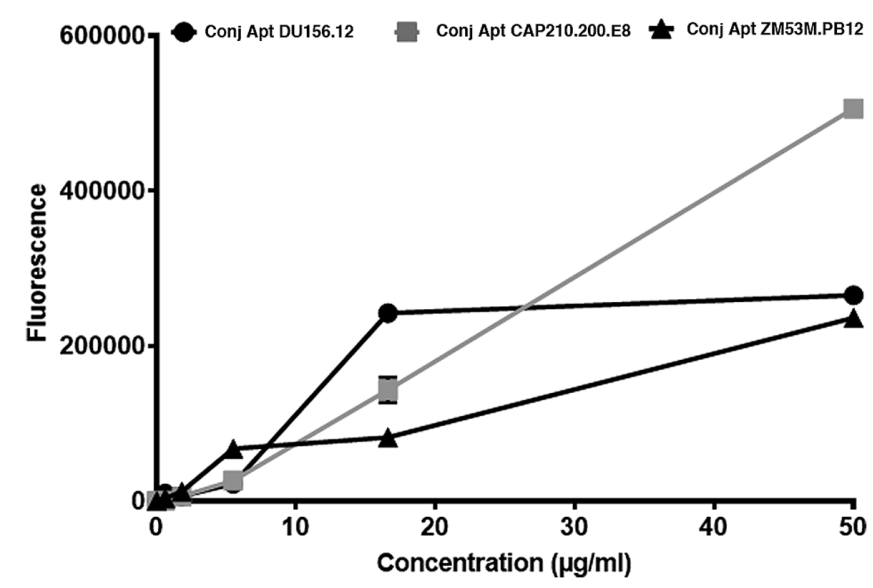

Figure 5. FITC-CSIR1.1 detection of captured HIV-1 particles. The detection of HIV-1 with aptamers was achieved by immobilizing the pseudoviruses on a solid surface with $10 \mathrm{E} 8 \mathrm{mAb}$. This was followed by the addition of FITC-conjugated CSIR 1.1 for fluorescence-based detection. HIV-1 subtype C DU156.12, CAP 210.E8 and ZM53M.PB12 pseudoviruses were used, and the experiment was repeated three times on different days. Data are presented as the mean \pm standard deviation of three replicate experiments repeated on different days. FITC, fluorescein isothiocyanate; conj, conjugated; apt, aptamer.

was a recombinant glycoprotein, whether FITC-CSIR1.1 could recognize the gp120 when expressed in its natural environment was assessed. To achieve this, a HIV-1 capture assay was performed (32) with three HIV-1 subtype C pseudoviruses, ZM53M.PB12, DU156.12 and CAP210.E8, known to interact with CSIR1.1 (10). The virus was captured with the $10 \mathrm{E} 8 \mathrm{mAb}(38)$ that binds gp41 and detected using different concentrations of the FITC-CSIR1.1 aptamer ranging from $0.00-0.05 \mathrm{mg} / \mathrm{ml}$. The choice of $10 \mathrm{E} 8 \mathrm{mAb}$ for capture was because it binds the membrane-proximal external region of the viral glycoprotein, thus making it less likely to compete with the aptamer for binding to the envelope (39). FITC-CSIR1.1 was observed to bind and detect all three viruses as expected (Fig. 5). When comparing capture of the viruses by the highest concentration of aptamer, CAP210.E8 pseudovirus was the most captured with a fluorescence intensity of 506,000 \pm 3.71 . The other two viruses, DU156.12 and ZM53M.PB12 had fluorescence intensities of $265,333 \pm 1.67$ and $236,333 \pm 1.47$, respectively. The P-value of the comparison of the three viruses' fluorescence intensities was 0.859 , suggesting that they were not significantly different.

Previous studies have shown that antibodies specific to gp120 are used in flow cytometry to detect HIV-1 infected cells (40). The fluorescence emission of the FITC-conjugated CSIR1.1 aptamer was therefore compared to a commercially available polyclonal biotin-conjugated anti-gp120 antibody using the capture assay, one of the three HIV-1 subtypes C mentioned above, ZM53M.PB12, was chosen for this experiment, given that statistically, all three viruses had similar fluorescence intensity. The same concentration of the aptamer and biotin-conjugated antibody was used. However, this antibody had to be detected with FITC-conjugated streptavidin. Thus, the FITC-streptavidin/biotin-antibody interaction was first optimized; the strongest fluorescence signal was detected when the FITC-streptavidin was used at a dilution of 1:2,000 (Fig. S3). When the FITC-CSIR1.1 and the biotin-antibody were compared to detect the captured

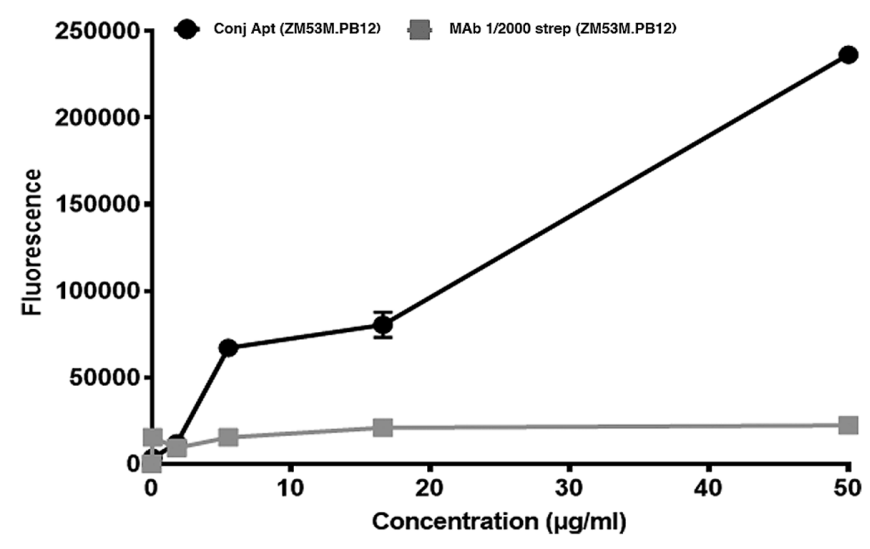

Figure 6. Comparison of the fluorescence signal emitted by the FITC-CSIR1.1 aptamer and FITC-streptavidin/biotin-anti-gp120. HIV-1 subtype C ZM53M. PB12 was captured with 10E8 mAb, followed by detection with the aptamer or the antibody. Data are presented as the mean \pm standard deviation of three replicate experiments repeated on different days. FITC, fluorescein isothiocyanate; conj, conjugated; apt, aptamer.

HIV-1, the fluorescence emitted by the aptamer was markedly stronger (Fig. 6). This difference was also observed to increase significantly as the concentrations of the aptamer and antibody were increased. For example, at $<20 \mu \mathrm{g} / \mathrm{ml}$ of both aptamer and antibody, the difference was $\sim 3$-fold. This increased to 8 -fold at $50 \mu \mathrm{g} / \mathrm{ml}$, where the aptamer produced an average fluorescence of $236,333 \pm 0.063$, while the antibody had an average of $22,333 \pm 1.53$. The difference between the aptamer and antibody was statistically significant $(\mathrm{P}<0.01)$ determined using an upraised t-test. Thus, FITC-CSIR1.1 performance was greater than that of the antibody destined for the same application.

HIV-1 neutralization with the FITC-conjugated CSIR1.1 aptamer. The CSIR1.1 aptamer has been shown to neutralize HIV-1 (10). HIV-1 neutralization efficiency of the conjugate was compared with the unconjugated (parent) aptamer to determine whether the conjugation of CSIR1.1 with FITC altered the aptamer's structure and/or functionality. CAP239. G3J, HIV-1 subtype C pseudovirus, known to be sensitive to CSIR1.1 (41), was used in the neutralization assay. As shown in Fig. 7, the inhibitory activity of both the conjugated and unconjugated aptamers was similar, judging by the consistency of inhibition. The P-value, determined using an unpaired t-test, was 0.784 ; therefore, the difference in inhibition between the two aptamers was not statistically significant. Thus, these data suggested that the conjugation with FITC did not alter the aptamer interaction with the viral envelope.

\section{Discussion}

Aptamers are nucleic acid molecules that can be synthesized to bind to various targets $(42,43)$. As a result, these molecules are being studied and synthesized as alternative reagents to antibodies in research, and for diagnosis and treatment of different diseases $(42,44)$. The present study investigated the possibility of converting previously isolated and studied aptamers against HIV-1 gp120 (10,11), fluorescence-labelled alternative reagents for use in applications requiring antibodies. It was shown that the CSIR1.1 aptamer could be conjugated efficiently to FITC fluorescent dye and used to detect gp120 in different assay formats 


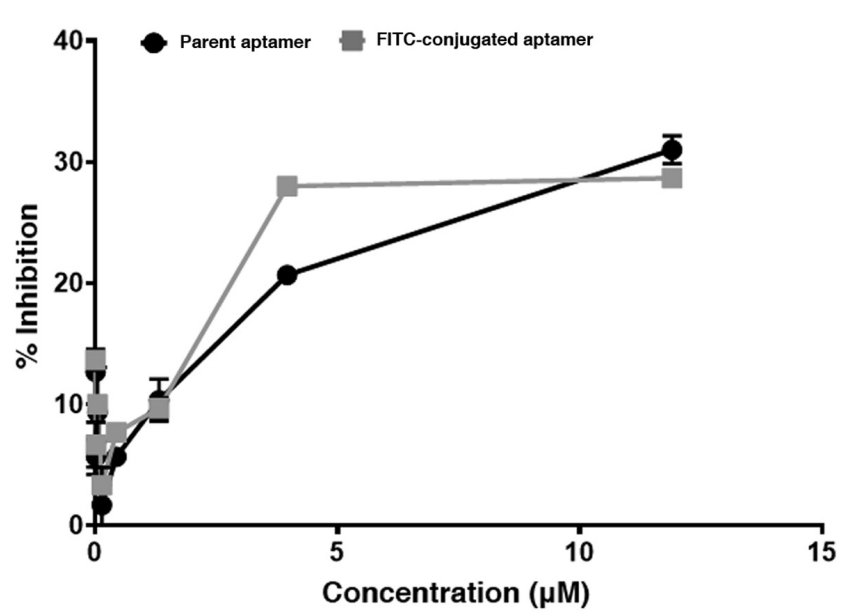

Figure 7. Comparison of the neutralizing effects of the CSIR1.1 parent aptamer and the FITC-conjugated aptamer to subtype C CAP239.G3J pseudovirus. The virus was incubated with CSIR1.1 parent aptamer (round symbol) or FITC-conjugated CSIR 1.1 (square symbol) before infection of TZM-bl cells. Data are presented as the mean \pm standard deviation of two different experiments repeated on two different days. FITC, fluorescein isothiocyanate.

involving flow cytometry and HIV capture assay. In addition, this fluorescence-labelled aptamer performed better than the antibody-based detection system designed for similar use. Given the low costs associated with synthesizing aptamers (11), it is suggested that FITC-CSIR1.1 is potentially a more viable alternative to antibodies for use in these applications.

The conjugation of FITC with the aptamers was more efficient with CSIR1.1 compared to UCLA1. This is likely due to the differences in the structure of the two aptamers. As shown in Fig. 8, CSIR1.1 has an amine group at its 5' end where it is expected to react with the carboxyl group of FITC. However, in UCLA1, there are extensive modifications at the 5 ' and $3^{\prime}$ ends that possibly prevent effective conjugation with FITC (25). To be more specific, the $5^{\prime}$ end of UCLA1 is modified with a dimethoxyltrityloxy- $\left(\mathrm{CH}_{2}\right)_{6}-\mathrm{SS}-\left(\mathrm{CH}_{2}\right)_{6}$-phospho linker, whereas its $3^{\prime}$ end is modified with an inverted dT for stability (11) (Fig. 8). Since these extensive modifications are at the sites where the aptamer is expected to bind FITC, these may be the cause of obstruction to the reaction, thus resulting in a decreased conjugation efficiency observed for this aptamer.

The fact that the FITC-CSIR1.1 neutralization of HIV-1 mirrored that of the unconjugated parent aptamer showed that the conjugation reaction did not alter the aptamer's interaction with gp120. This is very important, given that CSIR1.1 was shown in our laboratory to have high affinity and specificity for HIV-1 gp120 (10). It was essential that this property be maintained after the conjugation reaction if it is intended for use as an alternative to high affinity and high specificity molecules, such as antibodies $(45,46)$.

Infected and gp120 expressing cells and latex beads coupled to gp120 were used to investigate the possibility of using this aptamer to detect HIV-1 by flow cytometry. The FITC-CSIR1.1 molecule bound to the HIV-1 gp120 and could be detected by fluorescence. The $\sim 16 \%$ increase in fluorescence intensity observed by flow cytometry was significantly higher than the values obtained by other investigators using fluorescence-labelled antibodies against gp120. For example, when $\mathrm{H} 9$ cells were used to detect HIV by flow cytometry using

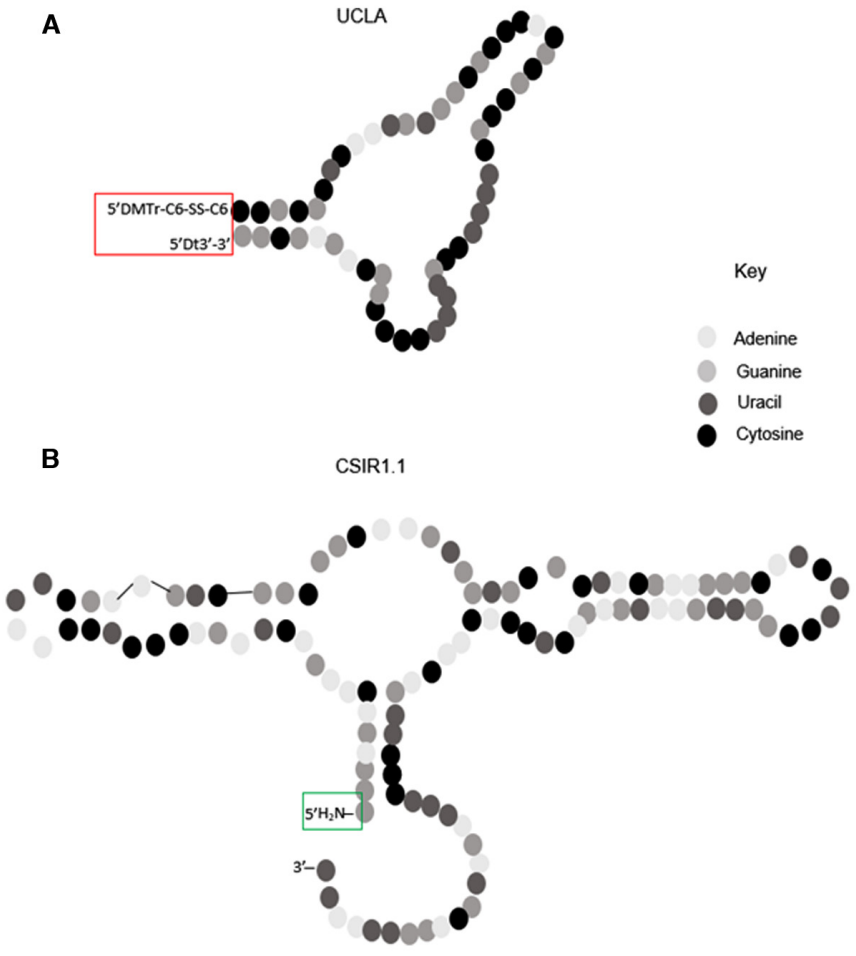

Figure 8. An illustration of the different structures and sequences of UCLA1 and CSIR1.1 aptamers. (A) Shows the structure of UCLA1 where the dimethoxyltrityloxy- $\left(\mathrm{CH}_{2}\right)_{6}-\mathrm{SS}-\left(\mathrm{CH}_{2}\right)_{6}$-phospho linker and inverted dT modifications are highlighted in red. (B) Shows the structure of CSIR1.1 aptamer with the reaction side highlighted in green. UCLA1 was adapted from Cohen et al (25) whereas CSIR1.1 was adapted from London et al (10).

gp120 monoclonal antibodies, the percentage fluorescence was reported to be 2.2 and $0.59 \%$ for infected and uninfected cells, respectively (47).

It is noteworthy that the use of FITC conjugated aptamers as flow cytometry reagents is not unprecedented. For example, a study by Rong et al (48) using a FITC-conjugated aptamer showed that this molecule bound specifically to cancer cells with no or little binding to non-cancerous cells. Similarly, Song et al (17) demonstrated that a single stranded DNA aptamer conjugated to FITC could differentiate target cancer cells from a mixture of cells in media using flow cytometry. Therefore, these data confirmed the potential of using this aptamer-based reagent to detect HIV-1 gp120 using flow cytometry and also showed that it could perform better than antibodies in some cases.

Given that aptamers are destined for use as alternatives in applications that usually require antibodies, it was important that FITC-CSIR1.1 be compared to an antibody-based assay. As shown by the capture assay, the signal emitted by the FITC-conjugated aptamer after binding gp120 expressed on HIV-1 envelope was markedly higher than that emitted using a FITC-conjugated streptavidin/biotin-antibody system. One possible explanation for this is that the size of antibodies, $150 \mathrm{kDa}(49)$, is much larger than that of the CSIR1.1 aptamer, at $55 \mathrm{kDa},(10)$. Thus, it is possible that fewer molecules of the antibody were bound on the surface of the virus compared to the aptamer, resulting in a stronger signal for the latter. The relatively small size of aptamers is a well-known advantage of these molecules over their competitors. It allows them to bind with high specificity and affinity to 
their targets. This was previously shown where aptamers could penetrate and be retained by tumor cells longer than antibodies, both in vitro and in vivo (50). As a result, they could be employed as molecular imaging probes (44).

Generally, a capture assay utilizes a minimum of two antibodies, one for capture and the other for detection (26). However, an added advantage of the aptamer capture assay is that if two aptamers that bind gp120 at different sites are selected (by SELEX), one aptamer will be used to capture the virus, and the other will be used for detection thus, completely removing the need for use of an antibody in the assay. Moreover, the flow cytometry data indicated that there was no need for use of an antibody together with the aptamer. Unlike antibodies, the cost of synthesizing aptamers has been greatly reduced through several years of modifications and refinements leading to production time of weeks compared to antibodies with longer in vivo procedures that span months $(8,11)$. Of note, the cost to perform a capture assay using a pair of gp120 specific antibodies is $~ 136$ USD per reaction compared to only 40 USD for a pair of aptamers, as performed in the present study, resulting in a 3 -fold cost reduction.

Despite the advantages of the aptamer-based method and potential ease of application in a hospital setting, the study does not come without limitations. For example, the FITC conjugated aptamer could have been tested against several HIV-1 strains to determine whether it retained its broad spectrum of binding against the virus (10). In addition, the FITC-aptamer duration of conjugation was not determined in the present study. It is important that the half-life of this bond be determined in future studies since chemical bonds are known to degrade; furthermore, fluorophores can be bleached, thus reducing or abrogating their signal $(51,52)$.

In conclusion, the results of the present study showed that the CSIR1.1 aptamer can be conjugated to FITC and used to detect gp120 by flow cytometry or HIV-1 capture assay. This reagent can potentially perform at a higher level than its antibody-based counterpart, employed in the same applications. More importantly, these findings support the view that aptamers can be used as cheaper, but equally, if not more suitable substitutes for antibodies. Such reagents will be beneficial for research-poor settings, especially in developing countries worldwide, where the cost of reagents is one of the biggest obstacles to research. They will also be valuable in diagnostics involving techniques such as ELISA and imaging. In addition, the high cost of producing antibodies in animals (7), and the inability to produce them against non-immunogenic targets (53), further supports the development of aptamer-based reagents.

\section{Acknowledgements}

We would like acknowledge the generous donation of HIV-1 envelope plasmids for the production of pseudoviruses by the AIDS Research Unit at the National Institute for Communicable Diseases (NICD).

\section{Funding}

This work was supported by the Council for Scientific and Industrial Research Parliamentary Grant (grant no. V1YAT74), and the Council for Scientific and Industrial Research Masters Studentship.

\section{Availability of data and materials}

The data sets used and/or analyzed during the present study are available from the corresponding authors on request.

\section{Authors' contributions}

KA and HM conceptualized and designed the study. KM performed the experiments and collected the data. KM, PNF, and KA analyzed and interpreted the data. KM wrote the draft manuscript. PNF, HM, KA supervised the findings and provided critical and intellectual revisions and feedback. HM, PNF and KA confirmed the authenticity of all the raw data. All authors revised the manuscript as well as read and approved the final version.

\section{Ethics approval and consent to participate}

Ethics approval for this study was obtained from the Council for Scientific and Industrial Research Ethics Committee (approval no. 184/2016) and the waiver from the Human Research Ethics (Medical) Committee (approval no. M1611138) of the University of the Witwatersrand. Albeit the study did not require the use of animal samples, both institutions require that ethics/ethics waiver be obtained prior to conducting any work.

\section{Patient consent for publication}

Not applicable.

\section{Competing interests}

The authors declare that they have no competing interests.

\section{References}

1. UNAIDS: Global HIV \& AIDS statistics - 2019 fact sheet. 2020.

2. World Health Organisation: Latest HIV estimates and updates on HIV policies uptake. In: Global HIV, Hepatitis and STI Programmes. 2020.

3. Zeng JY, Li X, Yuan HX, Ma ML, Li DD, Ma J and Liao S: Screening ssDNA aptamers against HIV P24 antigen using agarose beads as carriers. Bio Web Conf 8: 10, 2017.

4. Freed EO: HIV-1 gag proteins: Diverse functions in the virus life cycle. Virology 251: 1-15, 1998.

5. Wyatt R, Kwong PD, Desjardins E, Sweet RW, Robinson J, Hendrickson WA and Sodroski JG: The antigenic structure of the HIV gp120 envelope glycoprotein. Nature 393: 705-711, 1998.

6. Chan DC and Kim PS: HIV entry and its inhibition. Cell 93: 681-684, 1998.

7. Guo KT, Ziemer G, Paul A and Wendel HP: CELL-SELEX: Novel perspectives of aptamer-based therapeutics. Int J Mol Sci 9: 668-678, 2008.

8. Chen A and Yang S: Replacing antibodies with aptamers in lateral flow immunoassay. Biosens Bioelectron 71: 230-242, 2015.

9. Toh SY, Citartan M, Gopinath SC and Tang TH: Aptamers as a replacement for antibodies in enzyme-linked immunosorbent assay. Biosens Bioelectron 64: 392-403, 2015.

10. London GM, Mayosi BM and Khati M: Isolation and characterization of 2'-F-RNA aptamers against whole HIV-1 subtype C envelope pseudovirus. Biochem Biophys Res Commun 456: 428-433, 2015.

11. Mufhandu HT, Gray ES, Madiga MC, Tumba N, Alexandre KB, Khoza T, Wibmer CK, Moore PL, Morris L and Khati M UCLA1, a synthetic derivative of a gp120 RNA aptamer, inhibits entry of human immunodeficiency virus type 1 subtype C. J Virol 86: 4989-4999, 2012. 
12. Tuerk $\mathrm{C}$ and Gold L: Systematic evolution of ligands by exponential enrichment: RNA ligands to bacteriophage T4 DNA polymerase. Science 249: 505-510, 1990.

13. Walker LM, Phogat SK, Chan-Hui PY, Wagner D, Phung P, Goss JL, Wrin T, Simek MD, Fling S, Mitcham JL, et al; Protocol G Principal Investigators: Broad and potent neutralizing antibodies from an African donor reveal a new HIV-1 vaccine target. Science 326: 285-289, 2009.

14. Glökler J, Schütze T and Konthur Z: Automation in the high-throughput selection of random combinatorial libraries - different approaches for select applications. Molecules 15: 2478-2490, 2010.

15. Stoltenburg R, Reinemann C and Strehlitz B: SELEX - a (r) evolutionary method to generate high-affinity nucleic acid ligands. Biomol Eng 24: 381-403, 2007.

16. Rohloff JC, Gelinas AD, Jarvis TC, Ochsner UA, Schneider DJ, Gold L and Janjic N: Nucleic acid ligands with protein-like side chains: Modified aptamers and their use as diagnostic and therapeutic agents. Mol Ther Nucleic Acids 3: e201, 2014.

17. Song KM, Lee S and Ban C: Aptamers and their biological applications. Sensors (Basel) 12: 612-631, 2012.

18. Ulrich H, Martins AH and Pesquero JB: RNA and DNA aptamers in cytomics analysis. Cytometry A 59: 220-231, 2004

19. Osborne SE, Matsumura I and Ellington AD: Aptamers as therapeutic and diagnostic reagents: Problems and prospects. Curr Opin Chem Biol 1: 5-9, 1997.

20. Osborne SE, Volker J, Stevens SY, Breslauer KJ and Glick GD: Design, synthesis, and analysis of disulfide cross-linked DNA duplexes. J Am Chem Soc 118: 11993-12003, 1996.

21. Zhang H, Wang Z, Li XF and Le XC: Ultrasensitive detection of proteins by amplification of affinity aptamers. Angew Chem Int Ed 45: 1576-1580, 2006.

22. Pavski V and Le XC: Detection of human immunodeficiency virus type 1 reverse transcriptase using aptamers as probes in affinity capillary electrophoresis. Anal Chem 73: 6070-6076, 2001.

23. Cao Y, Dong H, Yang Z, Zhong X, Chen Y, Dai W and Zhang X: Aptamer-conjugated graphene quantum dots/porphyrin derivative theranostic agent for intracellular cancer-related microrna detection and fluorescence-guided photothermal/photodynamic synergetic therapy. ACS Appl Mater Interfaces 9: 159-166, 2017.

24. Moutsiopoulou A, Broyles D, Dikici E, Daunert S and Deo SK Molecular aptamer beacons and their applications in sensing, imaging, and diagnostics. Small 15: e1902248, 2019.

25. Cohen C, Forzan M, Sproat B, Pantophlet R, McGowan I, Burton D and James W: An aptamer that neutralizes R5 strains of HIV-1 binds to core residues of gp120 in the CCR5 binding site. Virology 381: 46-54, 2008.

26. Alexandre KB, Gray ES, Mufhandu H, McMahon JB Chakauya E, O'Keefe BR, Chikwamba R and Morris L: The lectins griffithsin, cyanovirin-N and scytovirin inhibit HIV-1 binding to the DC-SIGN receptor and transfer to CD4(+) cells. Virology 423: 175-186, 2012.

27. Koniev $\mathrm{O}$ and Wagner A: Developments and recent advancements in the field of endogenous amino acid selective bond forming reactions for bioconjugation. Chem Soc Rev 44: 5495-5551, 2015

28. Shapiro R, Ellis S, Hingerty BE and Broyde S: Effect of ring size on conformations of aromatic amine-DNA adducts: The aniline-C8 guanine adduct resides in the B-DNA major groove. Chem Res Toxicol 11: 335-341, 1998.

29. Castronovo M, Radovic S, Grunwald C, Casalis L, Morgante M and Scoles G: Control of steric hindrance on restriction enzyme reactions with surface-bound DNA nanostructures. Nano Lett 8 : 4140-4145, 2008

30. Xu F, Pellino AM and Knoll W: Electrostatic repulsion and steric hindrance effects of surface probe density on deoxyribonucleic acid (DNA)/peptide nucleic acid (PNA) hybridisation. Thin Solid Films 516: 8634-8639, 2008.

31. Yue Y, Li G, Yang Y, Zhang W, Pan H, Chen R, Shi F and Jin Y: Regulation of Dscam exon 17 alternative splicing by steric hindrance in combination with RNA secondary structures. RNA Biol 10: 1822-1833, 2013.

32. Alexandre KB, Gray ES, Lambson BE, Moore PL, Choge IA, Mlisana K, Karim SS, McMahon J, O'Keefe B, Chikwamba R, et al: Mannose-rich glycosylation patterns on HIV-1 subtype C gp120 and sensitivity to the lectins, Griffithsin, Cyanovirin-N and Scytovirin. Virology 402: 187-196, 2010.

33. Alexandre KB, Moore PL, Nonyane M, Gray ES, Ranchobe N, Chakauya E, McMahon JB, O'Keefe BR, Chikwamba R and Morris L: Mechanisms of HIV-1 subtype C resistance to GRFT, CV-N and SVN. Virology 446: 66-76, 2013.
34. Zhou J, Swiderski P, Li H, Zhang J, Neff CP, Akkina R and Rossi JJ: Selection, characterization and application of new RNA HIV gp 120 aptamers for facile delivery of Dicer substrate siRNAs into HIV infected cells. Nucleic Acids Res 37: 3094-3109, 2009.

35. Gray ES, Madiga MC, Hermanus T, Moore PL, Wibmer CK, Tumba NL, Werner L, Mlisana K, Sibeko S, Williamson C, et al; CAPRISA002 Study Team: The neutralization breadth of HIV-1 develops incrementally over four years and is associated with $\mathrm{CD}^{+} \mathrm{T}$ cell decline and high viral load during acute infection. J Virol 85: 4828-4840, 2011.

36. Lekkerkerker AN, Ludwig IS, van Vliet SJ, van Kooyk Y and Geijtenbeek TB: Potency of HIV-1 envelope glycoprotein gp120 antibodies to inhibit the interaction of DC-SIGN with HIV-1 gp120. Virology 329: 465-476, 2004.

37. Kojima T, Takei Y, Ohtsuka M, Kawarasaki Y, Yamane T and Nakano H: PCR amplification from single DNA molecules on magnetic beads in emulsion: Application for high-throughput screening of transcription factor targets. Nucleic Acids Res 33: e150, 2005.

38. Guttman M, Padte NN, Huang Y, Yu J, Rocklin GJ, Weitzner BD, Scian M, Ho DD and Lee KK: The influence of proline isomerization on potency and stability of anti-HIV antibody 10E8. Sci Rep 10: 14313, 2020.

39. Huang J, Kang BH, Pancera M, Lee JH, Tong T, Feng Y, Imamichi H, Georgiev IS, Chuang GY, Druz A, et al: Broad and potent HIV-1 neutralization by a human antibody that binds the gp41-gp120 interface. Nature 515: 138-142, 2014.

40. Rigg RJ, Dando JS, Escaich S, Plavec I and Böhnlein E: Detection of intracellular HIV-1 Rev protein by flow cytometry. J Immunol Methods 188: 187-195, 1995.

41. Grace Mothepane London: Isolation, Characterization and efficacy studies of 2'-F-RNA aptamers against HIV-1 subtype C enveloped pseudovirus. p292, 2013.

42. Wang YK, Zou Q, Sun JH, Wang HA, Sun X, Chen ZF and Yan YX: Screening of single-stranded DNA (ssDNA) aptamers against a zearalenone monoclonal antibody and development of a ssDNA-based enzyme-linked oligonucleotide assay for determination of zearalenone in corn. J Agric Food Chem 63: 136-141, 2015.

43. Torrini F, Palladino P, Brittoli A, Baldoneschi V, Minunni M and Scarano S: Characterization of troponin T binding aptamers for an innovative enzyme-linked oligonucleotide assay (ELONA). Anal Bioanal Chem 411: 7709-7716, 2019.

44. Xiang D, Zheng C, Zhou SF, Qiao S, Tran PH, Pu C, Li Y, Kong L, Kouzani AZ, Lin J, et al: Superior performance of aptamer in tumor penetration over antibody: Implication of aptamer-based theranostics in solid tumors. Theranostics 5: 1083-1097, 2015.

45. Margolis DM, Koup RA and Ferrari G: HIV antibodies for treatment of HIV infection. Immunol Rev 275: 313-323, 2017.

46. McSharry JJ: Analysis of virus-infected cells by flow cytometry. Methods 21: 249-257, 2000

47. McSharry JJ, Costantino R, Robbiano E, Echols R, Stevens R and Lehman JM: Detection and quantitation of human immunodeficiency virus-infected peripheral blood mononuclear cells by flow cytometry. J Clin Microbiol 28: 724-733, 1990.

48. Rong Y, Chen H, Zhou XF, Yin CQ, Wang BC, Peng CW, Liu SP and Wang FB: Identification of an aptamer through whole cell-SELEX for targeting high metastatic liver cancers. Oncotarget 7: 8282-8294, 2016

49. Singh AA, Pooe O, Kwezi L, Lotter-Stark T, Stoychev SH, Alexandra K, Gerber I, Bhiman JN, Vorster J, Pauly M, et al: Plant-based production of highly potent anti-HIV antibodies with engineered posttranslational modifications. Sci Rep 10: $6201,2020$.

50. Ying G, Zhu F, Yi Y, Chen J, Mei J, Zhang Y and Chen S: Selecting DNA aptamers for endotoxin separation. Biotechnol Lett 37: 1601-1605, 2015.

51. Bhatt N, Huang PJ, Dave N and Liu J: Dissociation and degradation of thiol-modified DNA on gold nanoparticles in aqueous and organic solvents. Langmuir 27: 6132-6137, 2011.

52. Zheng Q, Jockusch S, Zhou Z and Blanchard SC: The contribution of reactive oxygen species to the photobleaching of organic fluorophores. Photochem Photobiol 90: 448-454, 2014.

53. Jayasena SD: Aptamers: An emerging class of molecules that rival antibodies in diagnostics. Clin Chem 45: 1628-1650, 1999.

This work is licensed under a Creative Commons Attribution-NonCommercial-NoDerivatives 4.0 International (CC BY-NC-ND 4.0) License. 